Igor Sukhotnik · Nirit Mor-Vaknin

Robert A. Drongowski · Ines Miselevich

Arnold G. Coran · Carroll M. Harmon

\title{
Effect of dietary fat on early morphological intestinal adaptation in a rat with short bowel syndrome
}

Accepted: 7 November 2003 / Published online: 24 April 2004

(C) Springer-Verlag 2004

\begin{abstract}
Among factors promoting mucosal hyperplasia after bowel resection, long-chain fatty acids may have a special role. The purpose of the present study was to evaluate the effects of high-fat diet (HFD) on early intestinal adaptation in rats with short bowel syndrome (SBS). Male Sprague-Dawley rats underwent either a bowel transection with re-anastomosis (Sham rats) or $75 \%$ small bowel resection (SBS rats). Animals were randomly assigned to one of three groups: Sham rats fed normal chow (Sham-NC); SBS rats fed NC (SBS-NC); and SBS rats fed HFD (SBS-HFD). Rats were killed on days 3 or 14 . Body weight and parameters of intestinal adaptation (overall bowel and mucosal weight, mucosal DNA and protein, villus height, and crypt depth) were determined at time of killing. By day 3, SBS-HFD rats demonstrated higher duodenal and jejunal bowel and mucosal weights and ileal villus height and jejunal crypt depth vs SBS-NC rats. By day 14 SBS-HFD rats continued to demonstrate increased duodenal and jejunal bowel weight and duodenal mucosal weight vs SBS-NC animals. We conclude that early exposure to HFD both augmented and accelerated structural bowel adaptation in a rat model of SBS.
\end{abstract}

I. Sukhotnik · N. Mor-Vaknin · R. A. Drongowski

A. G. Coran · C. M. Harmon

Section of Pediatric Surgery,

C.S. Mott Children's Hospital and University

of Michigan Medical School,

Ann Arbor, MI, USA

I. Sukhotnik $(\bowtie) \cdot$ I. Miselevich

Rappaport Faculty of Medicine,

Technion, Bnai Zion Medical Center,

Haifa, Israel

E-mail: igor-dr@internet-zahav.net

Tel.: +972-4-8256815

Fax: + 972-4-8346083

Present address: I. Sukhotnik

Department of Surgery B, Carmel Medical Center,

7 Michal street, 34362 Haifa, Israel
Keywords Short bowel syndrome - Intestinal adaptation $\cdot$ Diet $\cdot$ Lipid

\section{Introduction}

The key to survival after massive small bowel resection is the ability of the residual intestine to adapt. In humans, intestinal adaptation begins within $48 \mathrm{~h}$ of resection and includes morphological and functional changes of the residual bowel $[1,2]$. Throughout the process of adaptation, the small bowel increases its absorptive surface area and its functional capacity in attempt to meet the body's metabolic and growth needs [3]. The specific signals for intestinal adaptation after bowel resection remain unclear; however, the observation that enteral nutrition stimulates this process is well documented [4, $5,6,7]$. In fact, it has been suggested that in the absence of enteral feeding adaptation does not occur $[4,5]$. The mechanism(s) whereby enteral nutrients influence intestinal adaptation following resection is uncertain. Research is now directed at those trophic factors that may in the future have therapeutic implications for patients with short bowel syndrome. It has been suggested that pectin, short-chain triglycerides, long-chain fatty acids (LCFA), and glutamine have important roles as proadaptive agents in experimental animals with short bowel syndrome [8]. Long-chain fatty acids appear to be very effective stimulators of intestinal adaptation, more than either medium-chain fats or carbohydrates [3]. Consequently, a mixture of long- and medium-chain fats is recommended to patients with short bowel syndrome; medium-chain fats because they are "tolerated" more easily, and LCFA because they are important in stimulating intestinal adaptation $[2,3]$. Over the past decade considerable research has focused on the identification of the relatively late effect of LCFA on bowel adaptation in animal models of SBS [9, 10, 11]; however, understanding the mechanisms by which mucosal growth may be stimulated by lipids in the early stages of intestinal adaptation could lead to important clinical applications. 
The present study was undertaken to explore the effects of a high-fat diet (HFD) on the adaptive changes in the mucosa in the first 2 weeks following resection in a rat model of SBS.

\section{Materials and methods}

Animals

Male rats weighing 240-260 g were kept in individual stainless steel cages and initially fed ad libitum with normal rat chow and tap water. The rats were randomly assigned to one of three groups: (a) Sham-operated rats fed normal chow (Sham-NC); (b) rats with SBS fed normal chow (SBS-NC); and (c) rats with SBS fed a HFD (SBS-HFD).

After $48 \mathrm{~h}$ of acclimation to the environment and $12 \mathrm{~h}$ fast, the rats were anesthetized with intraperitoneal sodium pentobarbital (45 mg/kg; Butler Company, Columbus, Ohio).

\section{Surgical procedure}

Using sterile techniques, the abdomen was opened using a midline incision. Thirty-two rats underwent $75 \%$ bowel resection. The mesenteric vessels to the distal part of small bowel were ligated, the mesentery was divided, and $75 \%$ of the small bowel was resected, preserving the vascular arcade and leaving about $5 \mathrm{~cm}$ of proximal jejunum and $10 \mathrm{~cm}$ of distal ileum. Intestinal continuity was restored by an interrupted end-to-end single layer anastomosis using $6 / 0$ silk sutures. Another 16 rats underwent a sham operation, whereby the intestine was cut at a point $15 \mathrm{~cm}$ proximal to the ileo-cecal valve and re-anastomosed without resection. For all operations, the abdominal cavity was closed in two layers with a running suture using 3/0 Dexon "S" Polyglycolic Acid (Davis and Geck, N.Y.). Antimicrobial cream with pramoxine $\mathrm{HCl}$ (Mycitracin Plus, Johnson and Johnson) was applied to the incision for topical pain relief.

\section{Experimental model}

The animals recovered from the anesthetic and were allowed immediate access to water, although food was withheld until the following day. Experimental group animals were fed either normal chow $(10 \% \mathrm{kcal}$ fat $)$ or HFD (50\% kcal fat; Table 1).

\section{Intestinal adaptation analysis}

The animals were killed on days 4 or 15 postoperatively ( 3 or 14 of specific diet intake). The small intestine from the pylorus to the ileo-cecal valve was removed and divided into three segments: duodenum; jejunum to anastomosis; and terminal ileum. Portions of intestine $1 \mathrm{~cm}$ on either
Table 1 Composition of the various experimental diets

\begin{tabular}{lll}
\hline Ingredients & $\begin{array}{l}\text { Normal chow } \\
(\mathrm{gm} \%)\end{array}$ & $\begin{array}{l}\text { High-fat diet } \\
(\mathrm{gm} \%)\end{array}$ \\
\hline Casein, 80 Mesh & 200 & 200 \\
L-Cystein & 3 & 3 \\
Corn starch & 315 & 22.5 \\
Maltodextrin 10 & 35 & 125 \\
Sucrose & 350 & 147.5 \\
Cellulose, BW200 & 50 & 50 \\
Soybean oil & 25 & 25 \\
Lard & 20 & 200 \\
Minerals & 45 & 45 \\
Vitamins & 12 & 12 \\
Total: & & \\
Fat gm\%/ kcal\% & $4.3 / 10$ & $27.1 / 49.9$ \\
\hline
\end{tabular}

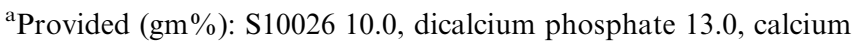
carbonate 5.5 , potassium citrate 16.5

${ }^{\mathrm{b}}$ Supplied (mg\%): vitamin mix V10001 10.0; choline bitartrats 2.0.

side of the anastomosis were discarded because of expected surgically induced hyperplasia occurring in this region. Each segment was weighed and the weight per centimeter of bowel length was calculated. The bowel was cut longitudinally, and the circumference was measured at three equidistant places as described by Dowling and Booth [12]. Surface area of the intestinal segment was calculated as the circumference was multiplied by the segment length and expressed per centimeter of bowel length. Mucosa was scraped from the underlying tissue and was weighed. Mucosal samples were homogenized using Kontes Tenbroeck Tissue Grinder. DNA and protein were extracted using TRIZOL reagent as described by of Chromozinski [13]. Histological sections were prepared from the jejunal and ileal remnants. The samples of intestinal tissues were fixed in a $10 \%$ formaldehyde solution $(2-3 \%$ methanol), then were embedded in paraffin wax using standard techniques. Sections ( $5 \mu \mathrm{m}$ each) were cut and stained with hematoxylin and eosin. The villus height and crypt depth for each specimen were measured, using an objective mounted micrometer (100× magnification) and an optical microscope $(10 \times 100$ magnification). Villus height and crypt depth data are from six rats, and each measurement consisted of the mean of five villi and crypts.

\section{Statistical evaluation}

The data are expressed as the mean \pm SEM. Statistical significance was determined by Student's $t$ test with a $p$ value $<0.05$ considered significant.

\section{Results}

\section{Body weight}

The sham-operated control rats gained weight immediately following the operation, whereas SBS rats maintained constant weight for 3 days (Fig. 1). Thereafter, 


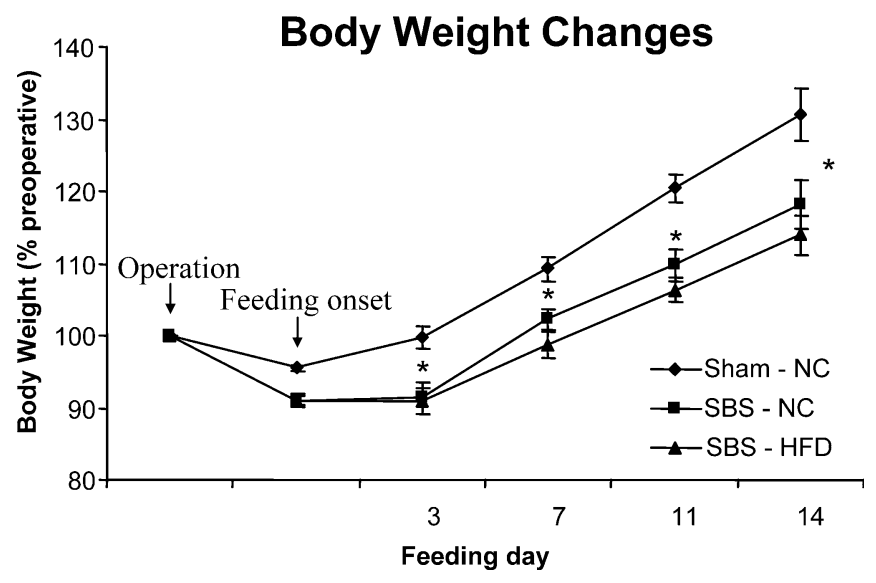

Fig. 1 Body weight changes (expressed as \%) of preoperative weight (mean \pm SEM) in control (Sham) and resected rats fed normal chow and high-fat diet (HFD). $S B S$ short bowel syndrome, $N C$ normal chow. $* p<0.05$ SBS vs Sham-NC rats, $\dagger p<0.05$ SBSHFD vs SBS-NC rats

both groups of the SBS rats fed different diets gained at approximately the same rate throughout the 2-week observation period; however, resected rats had significantly lower body weights than their sham-operated control counterparts. There was no statistical difference in the weights between SBS-NC and SBS-HFD animals.

\section{Bowel surface area}

On inspection, there was enlargement of the residual small intestine after bowel resection in both groups fed different diets. Compared with Sham-NC rats, SBS-NC rats had significantly larger surface area per unit of intestinal length in duodenum and jejunum on day 3 and in all segments on day 14 (Fig. 2). Early exposure to

\section{Mucosal Surface Area}

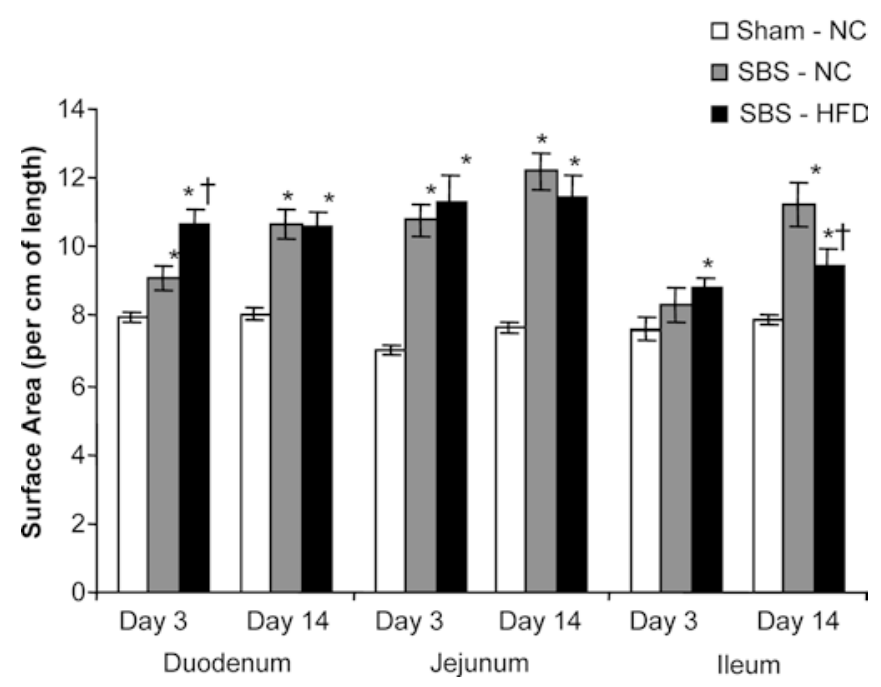

Fig. 2 Bowel surface area (expressed as $\mathrm{cm}^{2} / \mathrm{cm}$ length) in Sham, SBS-NC, and SBS-HFD rats. Values are mean \pm SEM. $S B S$ short bowel syndrome, $N C$ normal chow, HFD high-fat diet. ${ }^{*} p<0.05$ SBS vs Sham-NC rats, $\dagger p<0.05$ SBS-HFD vs SBS-NC rats
HFD led to an additional $17 \%$ increase in duodenal surface area on day 3; however, surface area of the jejunum did not change significantly but decreased in the ileum on day 14 compared with SBS-NC rats.

Total intestinal and mucosal weights

Overall total intestinal weights expressed as grams per centimeters of length per $100 \mathrm{~g}$ body weight significantly increased in all three segments in SBS-NC rats on day 3 and remained increased on day 14 compared with ShamNC animals (Fig. 3). The SBS-HFD rats showed higher duodenal $(39 \%)$ and jejunal $(25 \%)$ intestinal weights on days 3 and 14 compared with SBS-NC rats. Mucosal weights showed similar changes. Resected animals receiving normal chow showed greater mucosal weight per centimeter of bowel in all segments on days 3 and 14 compared with Sham-NC animals (Fig. 4). Early exposure to HFD led to an additional increase in mucosal weights in duodenum $(63 \%)$ and jejunum $(33 \%)$ on day 3 and in duodenum (26\%) on day 14 compared with SBS-NC animals.

\section{Mucosal DNA and protein}

The SBS-NC rats had significantly higher mucosal DNA levels in duodenum on day 3 and in all segments on day 14 compared with Sham-NC animals (Fig. 5). Following exposure to HFD, SBS rats demonstrated a significant increase in mucosal DNA in all segments on days 3 and 14 compared with Sham-control animals. Mucosal protein expressed per centimeter of length and per $100 \mathrm{~g}$ body weight was significantly higher in all segments on

\section{Bowel Weight Changes}

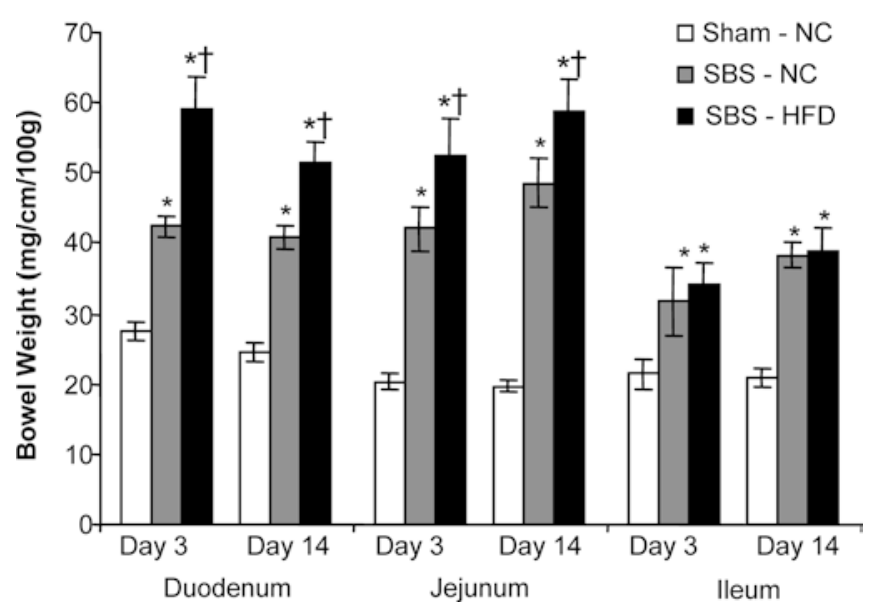

Fig. 3 Effect of bowel resection and dietary lipid on overall bowel weight (expressed as $\mathrm{mg} / \mathrm{cm}$ length/ $100 \mathrm{~g}$ body weight). Values are mean \pm SEM. $S B S$ short bowel syndrome, $N C$ normal chow, $H F D$ high-fat diet. $* p<0.05$ SBS vs Sham-NC rats, $\uparrow p<0.05$ SBS-HFD vs SBS-NC rats 


\section{Mucosal Weight Changes}

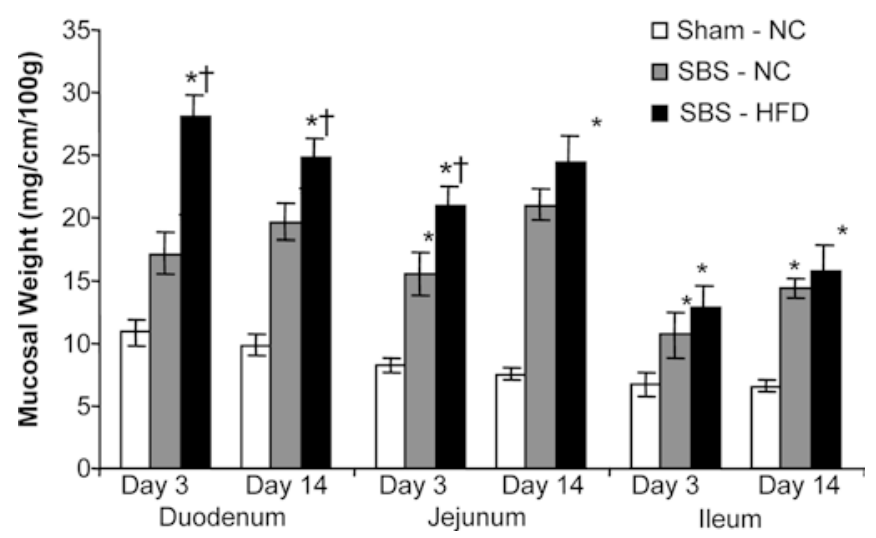

Fig. 4 Effect of bowel resection and dietary lipid on mucosal weight (expressed as $\mathrm{mg} / \mathrm{cm}$ length/ $100 \mathrm{~g}$ body weight). Values are mean \pm SEM. $S B S$ short bowel syndrome, $N C$ normal chow, $H F D$ high-fat diet. ${ }^{*} p<0.05$ SBS vs Sham-NC rats, $\dagger p<0.05$ SBS-HFD vs SBS-NC rats

day 3 and in jejunum and ileum on day 14 in SBS-NC rats compared with Sham counterparts (Fig. 6). The SBS-HFD rats showed a significant increase in mucosal DNA in all segments on days 3 and 14 compared with Sham-control animals. Additionally, SBS-HFD rats demonstrated higher mucosal DNA and protein mean values in duodenum and jejunum compared with SBS$\mathrm{NC}$ animals; however, these differences were not statistically significant.

\section{Histological findings}

In small bowel resected rats fed normal chow, ileal and jejunal villus height and crypt depth significantly in-

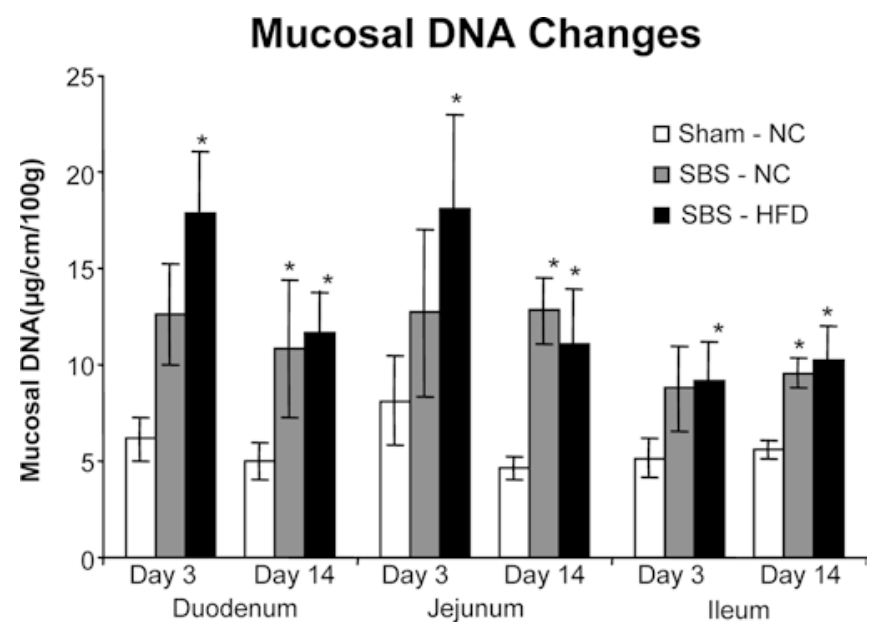

Fig. 5 Mucosal DNA content (expressed as $\mu \mathrm{g} / \mathrm{cm}$ length/ $100 \mathrm{~g}$ body weight) in Sham, SBS-NC, and SBS-HFD rats. Values are mean \pm SEM. SBS short bowel syndrome, $N C$ normal chow, $H F D$ high-fat diet. $* p<0.05$ SBS vs Sham-NC rats, $\dagger p<0.05$ SBS-HFD vs SBS-NC rats

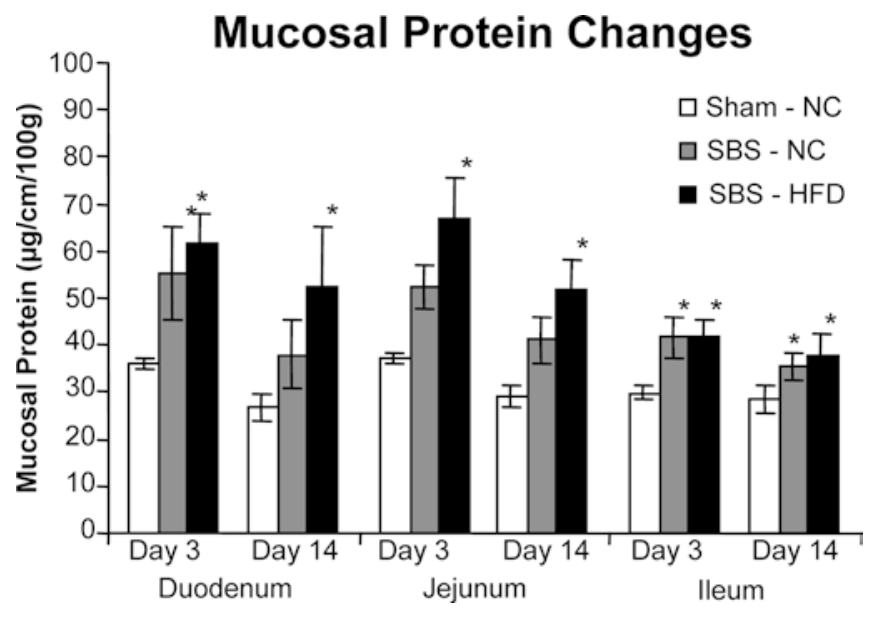

Fig. 6 Mucosal protein content (expressed as $\mu \mathrm{g} / \mathrm{cm}$ length/ $100 \mathrm{~g}$ body weight) in sham, SBS-NC, and SBS-HFD rats. Values are mean \pm SEM. $S B S$ short bowel syndrome, $N C$ normal chow, $H F D$ high-fat diet. ${ }^{*} p<0.05$ SBS vs Sham-NC rats, $\dagger p<0.05$ SBS-HFD vs SBS-NC rats

creased on days 3 (Fig. 7) and 14 (Fig. 8) compared with Sham-NC animals. The SBS-rats fed HFD showed significantly increased (compared with Sham-NC animals) villus height in jejunum and ileum and crypt depth in jejunum on day 3 , and villus height and crypt depth in jejunum and ileum on day 14. Additionally, SBS-HFD rats demonstrated significantly higher ileal villus height and jejunal crypt depth on day 3 and jejunal and ileal villus height on day 14 compared with SBS-NC animals.

\section{Discussion}

The importance of enteral nutrition in the adaptation of the small intestine after resection has been confirmed by

\section{Microscopic Bowel Appearance- Day 3}

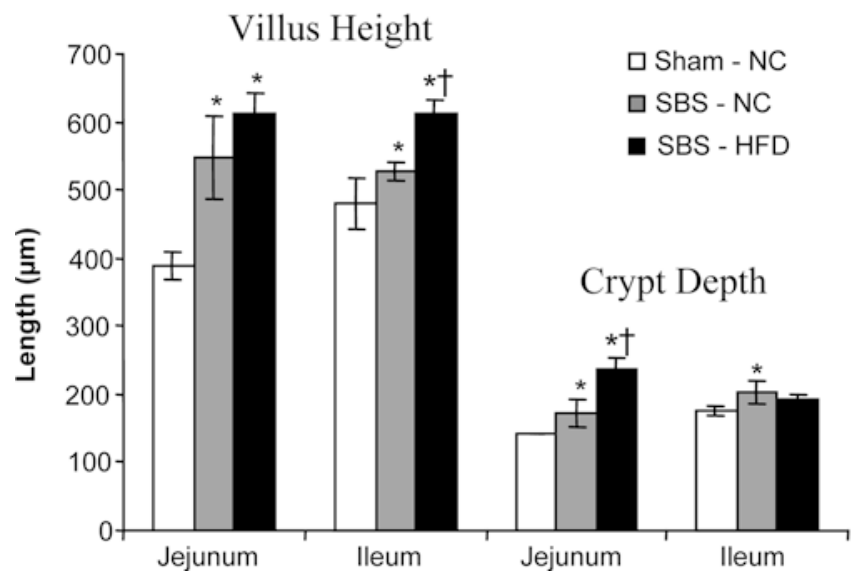

Fig. 7 Effect of bowel resection and dietary lipid on the microscopic appearance of the rat small intestine on day 3 following bowel resection. Values are mean \pm SEM. $S B S$ short bowel syndrome, $N C$ normal chow, HFD high-fat diet. * $p<0.05$ SBS vs Sham-NC rats, $\dagger p<0.05$ SBS-HFD vs SBS-NC rats 
Microscopic Bowel Appearance- Day 14

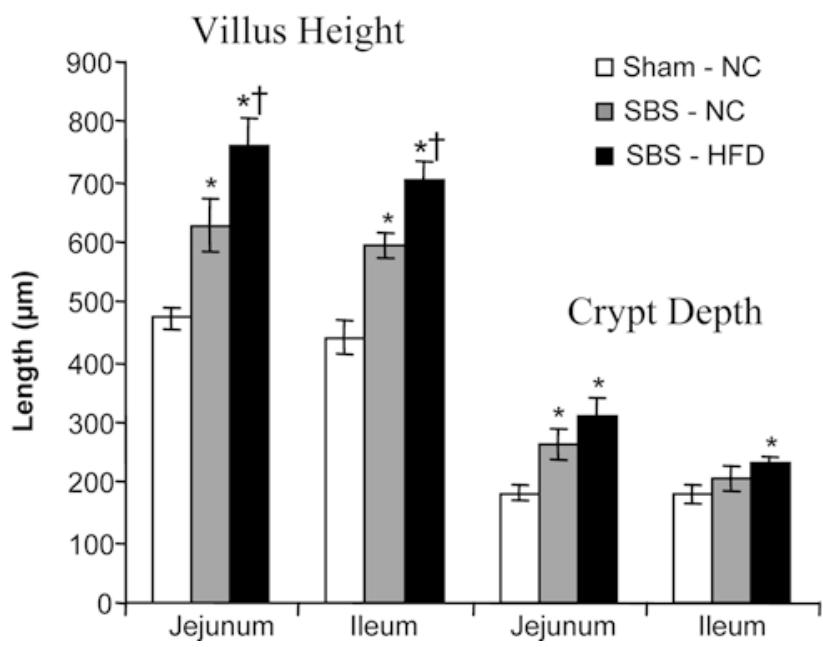

Fig. 8 Effect of bowel resection and dietary lipid on the microscopic appearance of the rat small intestine on day 14 following bowel resection. Values are mean $\pm \mathrm{SEM}$. $S B S$ short bowel syndrome, $N C$ normal chow, $H F D$ high-fat diet. ${ }^{*} p<0.05$ SBS vs Sham-NC rats, $\dagger p<0.05$ SBS-HFD vs SBS-NC rats

many studies $[4,6,7,14,15]$; however, the mechanism whereby food induces this adaptation is unknown. It is likely that enteral nutrition works through a number mechanisms, including direct stimulatory effects on the epithelial cells themselves, and through stimulation of secretion of trophic hormones from the gastrointestinal tract [3]. Although oral food intake is important for the observed response, the composition of the diet plays a major role in the adaptation process [16]. For example, it has been revealed that long-chain fatty acids appear to be more effective stimulators of intestinal adaptation than are either medium-chain fats or carbohydrates [3]. Several reports described significant trophic effects of long-chain fatty acids on small intestinal mucosa in normal animals $[15,16]$ and following bowel resection $[9,10,11]$. Experimental evidence in rats indicates that dietary linoleic acid can stimulate post-resection mucosal hypertrophy [10]. It also has been reported that animals with SBS receiving an essential fatty-acid-deficient diet have significantly impaired intestinal mucosal hyperplasia [9]. Little information is available concerning the influence of a HFD on intestinal adaptation in the very early postoperative period following bowel resection, and no recommendations have been given concerning the onset of a HFD as treatment for SBS. If intestinal adaptation begins within $48 \mathrm{~h}$ of resection and long fatty acids are one of the major factors in stimulating gut re-growth, then they should be used as early as possible. On the other hand, long-chain saturated fats are the least well tolerated in short bowel syndrome patients. To understand the mechanisms by which mucosal growth is stimulated by long-chain fatty acids in the initial steps of intestinal adaptation, we studied the effects of HFD on intestinal adaptation throughout the first 2 weeks following surgery. Intestinal re-growth after 3 and 14 days of dietary treatment was compared. We showed that in the rat model of SBS, animals fed normal chow showed initial signs of mucosal hyperplasia on day 3. After 14 days, all indices of intestinal adaptation (intestinal wall and mucosal weights, mucosal DNA, and protein) were significantly greater than those of control animals. The macroscopic appearance of the intestine and increased surface area per unit of bowel length suggests some degree of intestinal dilatation. Partial obstructive effects may explain the small bowel enlargement in the jejunum; however, the significant increase in surface area of the remnant of ileum seen on day 14 must be considered an indirect measure of a hypertrophic response.

Early exposure to HFD did not significantly change surface area compared with SBS-NC rats. At the same time, a significant increase in overall intestinal wall and mucosal weights was observed. These findings suggest that small bowel enlargement stimulated by HFD may be predominantly due to mucosal hyperplasia. The present results are consistent with data described by other investigators concerning the stimulating effect of dietary lipids on intestinal adaptation. We have shown that with exposure to a HFD this effect occurs significantly earlier than in rats fed normal chow.

The histopathological findings suggest that an initial increase in villus height was observed in SBS-NC rats in jejunum and ileum on day 3; however, maximal levels occurred on day 14. Early exposure to a HFD had a significant trophic effect on the small intestinal mucosa. The SBS-HFD rats showed increased villus height in the jejunum and ileum on days 3 and 14 compared with control group. Additionally, SBS-HFD rats had significantly higher ileal villus height and jejunal crypt depth on day 3 and ileal and jejunal villus heights on day 14 compared with SBS-NC animals.

Early exposure to a HFD has been shown to have beneficial effects on the small intestinal mucosa following bowel resection in rats. Early administration of HFD may be a useful adjunct in the management of children with short bowel syndrome, because its use has been shown to both accelerate and augment gut mucosal recovery and growth after massive small bowel resection.

\section{References}

1. Taylor SF, Sokol RJ (1995) Infants with short bowel syndrome. In: Hay WW (ed) Neonatal nutrition and metabolism. MosbyYear Book, St. Louis, pp 432-450

2. Biller JA (1987) Short bowel syndrome. In: Grand RI, Sutphen JL, Dietz WH (eds) Pediatric nutrition. Theory and practice. Butterworth, Stoneham, pp 481-487

3. Vanderhoof JA (1996) Short bowel syndrome. Neonatal Gastroenterol 23:377-386

4. Menge H, Grafe M, Lorenz-Meyer H, Riecken EO (1975) The influence of food intake on the development of structural and functional adaptation following ileal resection in the rat. Gut $16: 468-472$ 
5. Wesser E (1979) Nutritional aspects of malabsorption: short gut adaptation. Am J Med 67:1014-1019

6. Wesser E, Hernandez MH (1971) Studies of small bowel adaptation after intestinal resection in the rats. Gastroenterology 60:69-75

7. Buts JP, Morin CL, Ling V (1979) Influence of dietary components on intestinal adaptation after small bowel resection in rats. Clin Invest Med 2:59-66

8. Booth IW, Lander AD (1998) Short bowel syndrome. Bailliere's Clin Gastroenterol 12:739-772

9. Hart MH, Grandjean CJ, Park JHY, Erdman SH, Vanderhoof JA (1988) Essential fatty acid deficiency and postresection mucosal adaptation in the rats. Gastroenterology 94:682-687

10. Park JHY, Grandjean CJ, Hart MH, Vanderhoof JA (1989) Effects of dietary linoleic acid on mucosal adaptation after small bowel resection. Digestion 44:57-65

11. Vanderhoof JA, Park JHY, Herrington MK (1994) Effects of dietary menhaden oil on mucosal adaptation after small bowel resection in rats. Gastroenterology 106:94-99
12. Dowling RH, Booth CC (1967) Structural and functional changes following small intestinal resection in the rats. Clin Sci 32:139-149

13. Chromozinski P (1993) A reagent for the single-step simultaneous isolation of RNA, DNA and proteins from cell and tissue samples. BioTechniques 15:532-536

14. Levine GM, Deren JJ, Yezdimir E (1976) Small-bowel resection: oral intake is the stimulus for hyperplasia. Digest Dis 21:542-546

15. Williamson RCN, Chir MB (1978) Intestinal adaptation: mechanisms of control. N Engl J Med 298:1444-1450

16. Grey VL, Garofalo C, Greenberg GR, Morin CL (1984) The adaptation of the small intestine after resection in response to free fatty acids. Am J Clin Nutr 40:1235-1242 